\title{
Design and Health Evaluation of Monitoring Test Platform for Metro Traction Power Supply System
}

\author{
Hengchao Zheng and Yuhua Wang (iD \\ School of Electricity and Engineering, Nanjing Institute of Railway Technology, Nanjing, Jiangsu 210031, China \\ Correspondence should be addressed to Yuhua Wang; wangyuhua@njrts.edu.cn
}

Received 6 January 2021; Revised 5 February 2021; Accepted 22 February 2021; Published 5 March 2021

Academic Editor: Yi-Zhang Jiang

Copyright (C) 2021 Hengchao Zheng and Yuhua Wang. This is an open access article distributed under the Creative Commons Attribution License, which permits unrestricted use, distribution, and reproduction in any medium, provided the original work is properly cited.

\begin{abstract}
Subway traction power supply system is the energy source of subway operation. The normal operation of subway traction power supply system is directly related to the stable operation and safety of subway. If the subway traction power supply system breaks down, it not only will cause economic losses, but also may cause social disorder. Therefore, it is of great significance to study the traction power supply system of subway to ensure the safe operation of subway. The establishment of a reasonable monitoring and testing platform, the simulation analysis of the traction power supply system, and the regular health assessment can provide a strong guarantee for the safe operation of the subway. This paper aims to design a practical test platform through MATLAB modeling, so as to establish a simulation model of subway traction power supply system, and comprehensively monitor and grasp the health status of subway traction power supply system. This paper quantitatively evaluates the health status of subway traction power supply system, applies the principle of multilayer immunity, establishes the traction power supply system of analytic hierarchy process (AHP), and constructs the immune monitoring system of each layer. This method can provide the health status information of subway traction power supply system, subsystems, and components comprehensively, accurately, and intuitively and determine the subsystems and components that need to be maintained.
\end{abstract}

\section{Introduction}

The energy of subway operation comes from the subway traction power supply system, so the normal and safe operation of subway must be guaranteed by the subway traction power supply system. If something goes wrong with the subway traction power system, the subway system will not be able to provide power, which could throw the whole city into panic and chaos. Moreover, in case of a breakdown, it would certainly bring a heavy blow to the economy of the whole city. Therefore, this paper can see the importance of studying and analyzing the subway traction power supply system, which is of great significance for maintaining the order and normal operation of the city.

Due to the irreplaceable importance of the traction power supply system, this makes research and analysis of the system also become meaningful. There are many and varied approaches to system analysis and research, but it is very necessary to find a more systematic and scientific research method, and system simulation is like this. Due to the influence of actual factors, the simulation method of dynamic simulation is bound to be restricted by hardware conditions and the size of the experiment space, which will limit the scale of simulation in this article, and indirectly affect our country's scientific research. The shortcomings of mixed mode and dynamic analog simulation are also very obvious; that is, they both require a lot of time and money to experiment repeatedly. And it will be limited by the space of the experimental environment, so this simulation method has been slowly banned by digital simulation. This is because, while electronic technology and computer software development technology are developing at the speed of light, digital simulation has also been qualitatively improved, and the simulation method has avoided most of the shortcomings of digital-analog mixing and dynamic simulation. The most critical safety of this method is greatly guaranteed. It can be 
seen that digital simulation must have a limited prospect in future system simulation analysis and research [1-3].

Nowadays, many countries in the world have built rail transit. While the subway speeds up and facilitates urban transportation, it is also a symbol of a city and even a country, marking that the technological development of a city and even a country has reached a higher level. However, the level of science and technology in each country is very different, and the human and social environment of each country will directly or indirectly affect the development of urban transportation in that country. With the subway playing an increasingly important role in urban traffic, more and more researchers around the world are carrying out scientific research and innovation on subway technology. Their research on subway technology generally includes the power supply mode and power supply mode of subway. The voltage level and pulse number of the substation rectifier have also been studied.

The subways all over the world basically use DC power supply. Because the power of subway vehicles is small and the power supply radius is not large, the voltage levels of subways in the world are mainly DC and DC600. Foreign scholars have done detailed research and analysis on the voltage level of subway power supply. A subway power supply voltage was made, and the study was based on the development trend and safety of the subway. The literature pointed out that if the power of the locomotive is the same, the current and the traction network voltage are inversely proportional, so if the voltage of the catenary increases, the traction current must also decrease. So this article can draw a conclusion that the traction current under the DC supply voltage must be greater than the current under the DC supply voltage [4-7].

The faults of the subway traction power supply system can be divided into two major parts, including abnormal operating conditions and various short-circuit faults. This paper studies and analyzes the fault characteristics of the traction power supply system, which will make this paper better protect the traction power supply system. So far, a large number of researchers in the world have studied and analyzed the fault characteristics of the system, mainly in the direction of the fault characteristics of the DC feeder. The research found that when the traction load is different or when using different power supply methods, you will find that the fault characteristics of the feeder have obvious differences. On the one hand, the reference establishes a mathematical model of the subway traction power supply system, and at the same time, it also gives detailed electrical parameters of the traction power supply loop in the system. References have made detailed calculations and discussions on the differences between the current when a short-circuit fault occurs at the remote end of the substation outlet and the current when a short-circuit fault occurs at the near end of the substation outlet. The literature separately discusses the characteristics of the locomotive in different operating states, including states such as starting and short-circuit failure. The literature makes a detailed analysis of the harmonic content of the subway power supply system [8-11].

In summary, scholars at home and abroad have done many researches and analyses in different directions in the simulation of subway traction power supply system, and many aspects have been studied more maturely, but there are still some areas that deserve improvement [12-16]. The common structure of subway traction system is shown in Figure 1 .

The second section is the introduction to metro traction power supply system, the third section explains the MATLAB-based subway traction power supply system monitoring test platform design, and the forth section is the comprehensive health assessment of subway traction power supply system.

\section{Introduction to Metro Traction Power Supply System}

2.1. The Structure of the Subway Power Supply System. The entire subway is powered by the subway power supply system. From the perspective of different functions, the subway power supply system consists of six major systems: external power supply system, main substation, traction power supply system, stray current corrosion protection system, power monitoring system, and power lighting power supply system. The following is a brief introduction to each system.

External power supply: the external power supply of the subway power supply system is the city grid and is responsible for supplying power to the subway DC power supply system. External power supply generally includes centralized power supply, distributed power supply, and mixed power supply. Centralized power supply is a kind of external power supply which means that one main substation supplies centralized power to other subway substations. This is also the most commonly used method of subway in every city in our country. Distributed power supply is also a kind of external power supply mode, that is, direct use of urban medium-voltage power as the power supply of subway substation along the line. The main feature of this method is that it has multiple interfaces with the urban network and introduces multiple medium-voltage power sources directly from the urban network along the subway to supply power for the subway. However, the method is relatively independent and difficult to manage because of its complex structure. Hybrid power supply is a kind of power supply mode integrating centralized power supply and decentralized power supply. This method is seldom used in the cities of our country.

Traction power supply system: traction power supply system is the core part of subway power supply system, which occupies a very high position. The system is mainly composed of traction substation and traction network. The function of the system is to pass the high-voltage alternating current from the main substation through a series of functions such as voltage reduction and rectification to the subway locomotive to ensure the normal operation of the subway. The traction substation is the core part of the traction power supply system because; on the one hand, this part receives electrical energy from the external power source and a series of operations, such as step-down rectification, and it can convert the high-voltage AC power supply to the low-voltage DC power supply for output and 


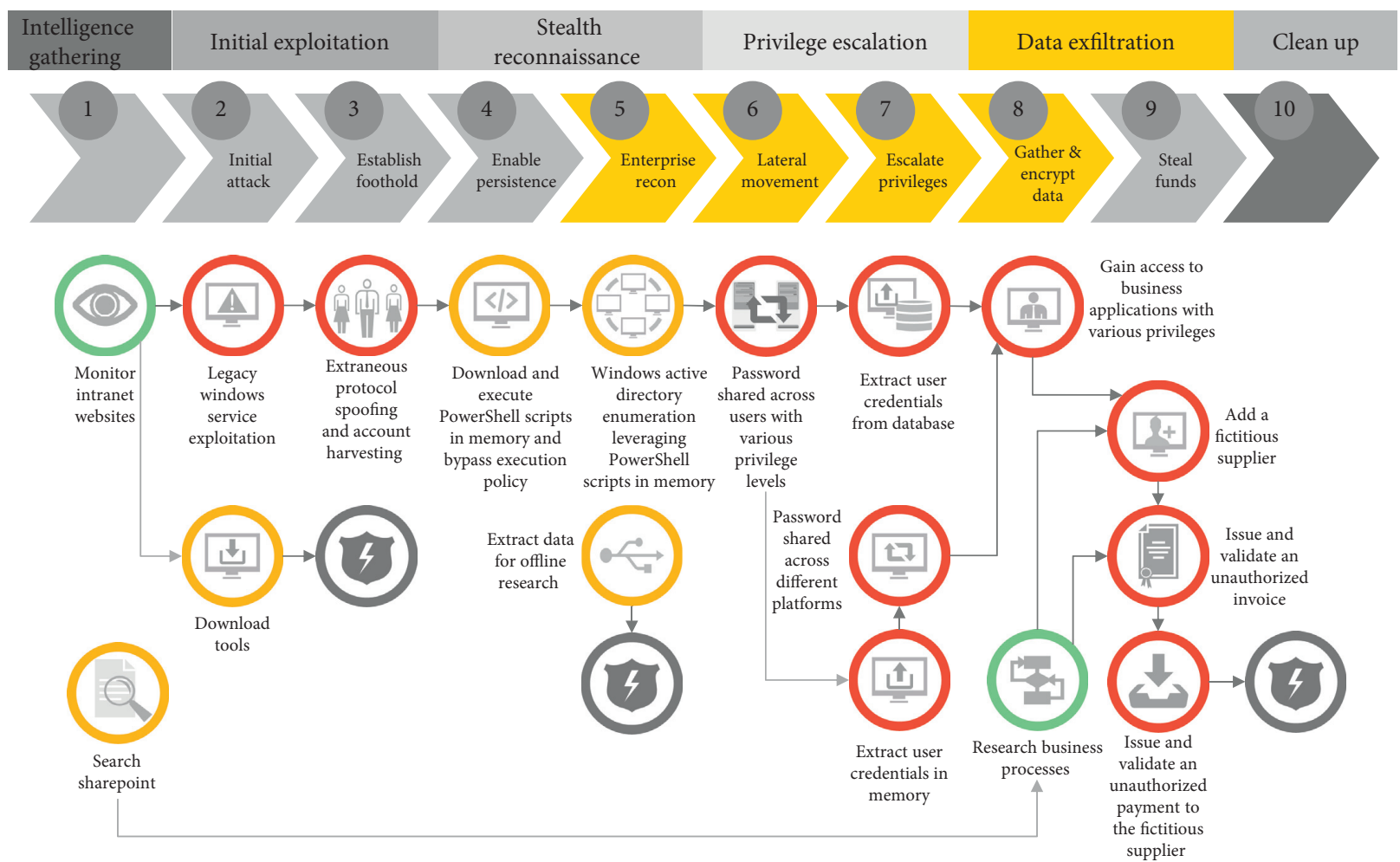

Figure 1: The common structure of subway traction system. The specific process is shown in title and subtitle.

transmission to the traction network. Considering the need to balance the output transformers of two units and reduce the influence on the system side harmonic current, nowadays, in my country, the equivalent pulsed traction rectifier units of two units are usually used to connect to the DC bus of the same part [17]. The schematic diagram of a relatively common traction power supply system is shown in Figure 2.

As can be seen from the above figure, the $\mathrm{AC}$ and DC switchgear, rectifier transformer, and rectifier in the figure together form the main wiring of the substation. The power supply lead-in wire and lead-out wire together form the main electrical wiring in the substation. The DC bus is the link between the traction substation and the traction network. The bus plays the role of linking up and down and gathering DC power and then redistributing it.

There are mainly two common DC bus wiring methods, namely, single- and double-bus wiring. The single-bus wiring method is more reliable and the structure is relatively simple, so it is more practical. In general, the wiring method will have two incoming lines and four feeders. Schematic diagram of the DC bus wiring methods is shown in Figure 3:

The third track mainly has the advantages of being neat and beautiful, small maintenance, and long service life. This method is mainly used when the DC voltage level is low. The overhead catenary mainly has the advantages of higher safety and easier separation of the pantograph. This method is more commonly used in systems with heavier traction loads and higher DC voltage levels. What kind of catenary should be chosen for each city is not fixed, and it is best to make a final decision after a comprehensive comparison based on the characteristics of the city.
Stray current corrosion protection system: in order to reduce the influence of the stray current generated by the DC power supply on the subway system and the surrounding environment, and further prevent the stray current from diffusing outward, the system may be different from other systems. It is very necessary to establish the stray current protection system in subway. In this way, the paper can monitor the stray current and corrosion protection of subway regularly or irregularly.

Power monitoring system: for better and safer operation of subway traffic, it is necessary to establish a real-time monitoring and data acquisition system for subway traffic on all parts of subway traffic, including substations and catenary equipment along the line. This system can manage, run, and schedule the subway traction power supply system through a series of operations [18].

\subsection{Construction Goals of the Subway Power Supply Traction} System. The subway traction power supply system is a very complex AC-DC hybrid system, and the subway locomotive load is a random and variable load, which will lead to the subway operation being not so simple. DC power has many advantages, so it is very reasonable to use DC power when subway is running. The advantages of this method mainly include the following: first, because there is no electric phase separation device, this paper does not need to consider the situation of the locomotive through electric phase separation, in order to ensure that the locomotive received by the electric energy is stable, reducing locomotive speed loss; secondly, the electromagnetic interference produced by DC 


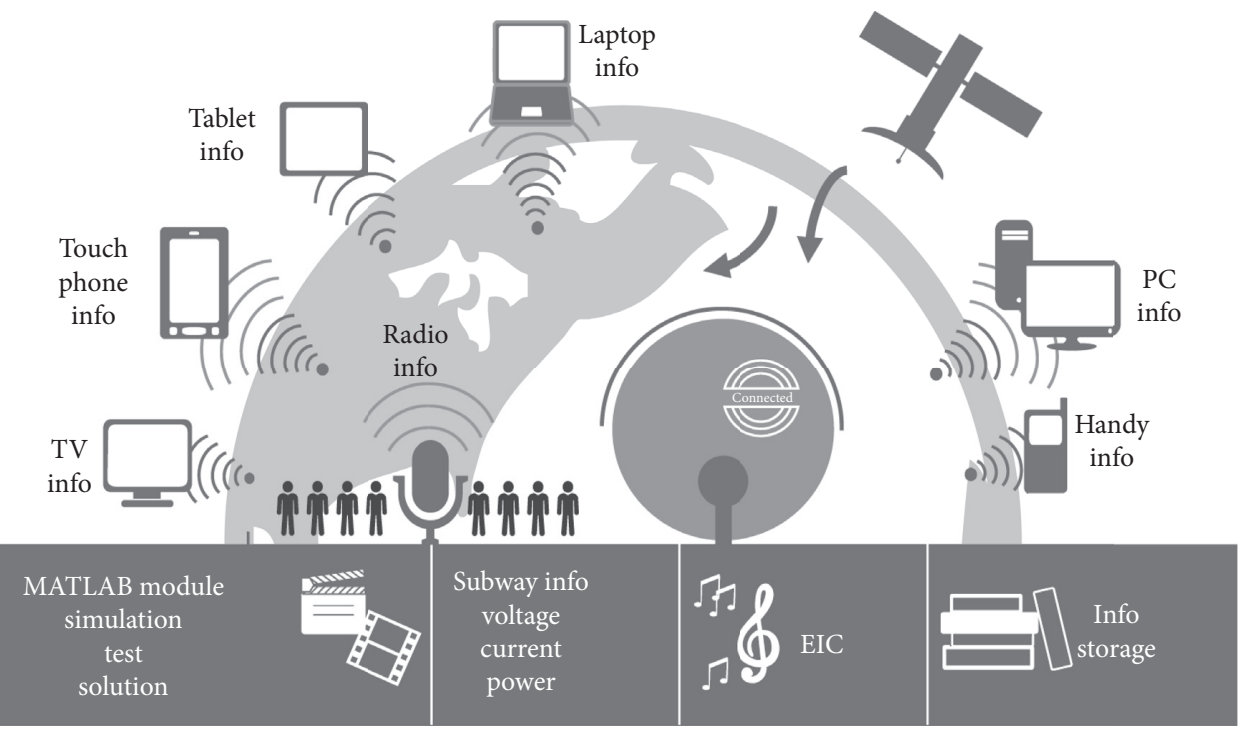

Figure 2: Common traction power supply system. The information is shown in the bottom.

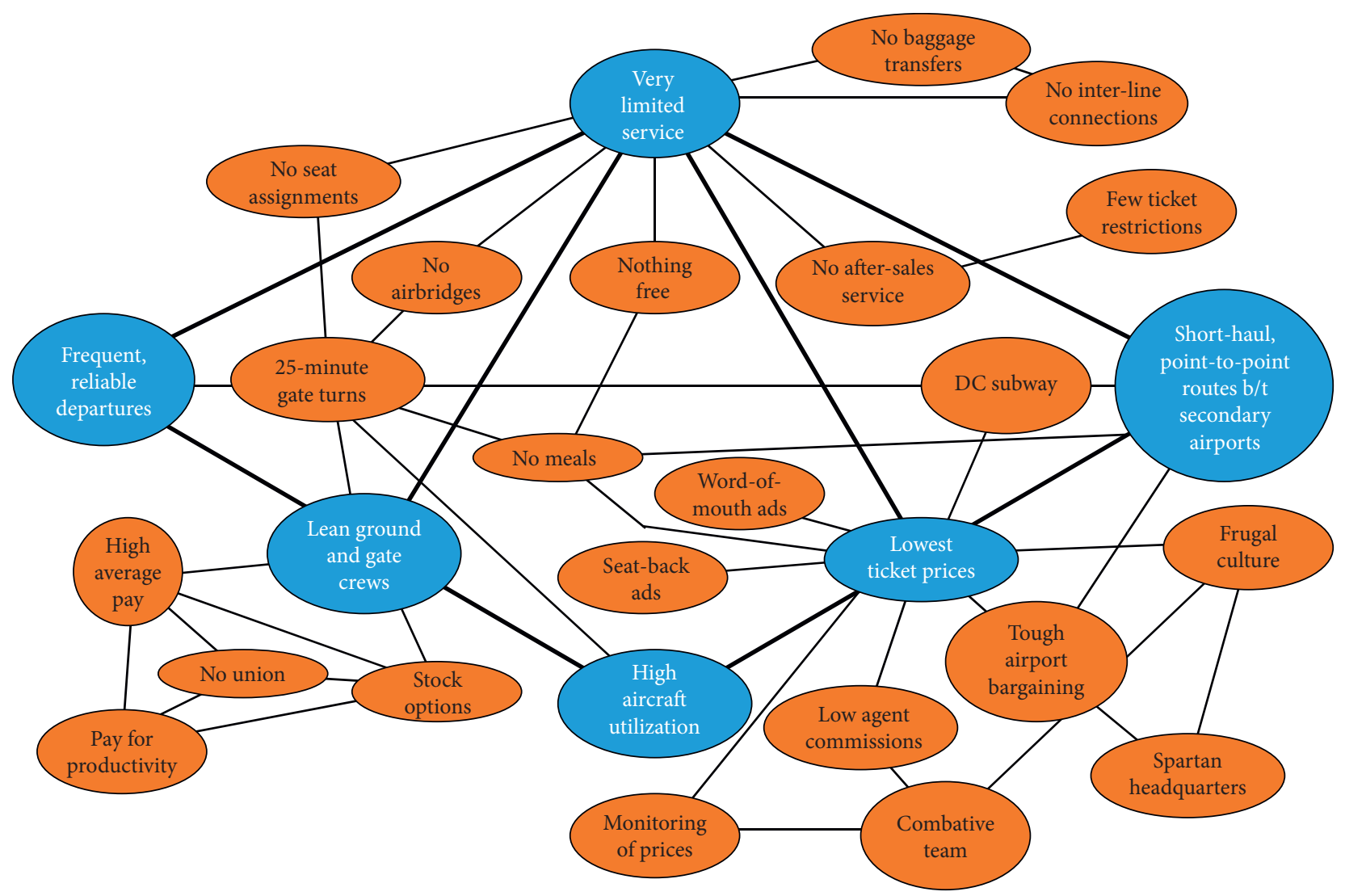

FIgURE 3: Schematic diagram of the DC bus wiring methods. The connection is shown between the lines.

traction power supply system is small. The adjacent power supply arms can be connected together so as to improve the power supply quality of the system. Meanwhile, the power harmonics generated in the system will be attenuated to a small extent.

In order to better model and analyze the traction power supply system of Chengdu Metro, this paper establishes a relatively complete traction power supply system model on the MATLAB platform. The model of traction power supply system established in this paper mainly includes five parts: external power supply model, traction substation model, DC bus model, catenary model, and rail and ground model. The overall flow of the three models is shown in Figure 4: 


\begin{tabular}{|c|c|c|c|c|c|c|c|c|c|c|c|}
\hline & Skill 1 & Skill 2 & Skill 3 & Skill 4 & Skill 5 & Skill 6 & Skill 7 & Skill 8 & Skill 9 & Skill 10 & \multirow{5}{*}{ Model unit 1} \\
\hline Staff 1 & $\mathrm{~L}$ & $\mathrm{~L}$ & M & M & $\mathrm{L}$ & $\mathrm{H}$ & $\mathrm{L}$ & $\mathrm{L}$ & $\mathrm{L}$ & $\mathrm{L}$ & \\
\hline Staff 2 & $\mathrm{~L}$ & M & M & M & $\mathrm{L}$ & $\mathrm{H}$ & $\mathrm{L}$ & $\mathrm{L}$ & $\mathrm{L}$ & M & \\
\hline Staff 3 & $\mathrm{~L}$ & M & M & M & $\mathrm{L}$ & $\mathrm{H}$ & $\mathrm{L}$ & $\mathrm{L}$ & M & $\mathrm{L}$ & \\
\hline Staff 4 & $\mathrm{~L}$ & $\mathrm{~L}$ & $\mathrm{~L}$ & $\mathrm{H}$ & M & $\mathrm{H}$ & $\mathrm{H}$ & $\mathrm{L}$ & $\mathrm{L}$ & $\mathrm{L}$ & \\
\hline Staff 5 & $\mathrm{~L}$ & $\mathrm{~L}$ & $\mathrm{~L}$ & $\mathrm{H}$ & $\mathrm{L}$ & $\mathrm{L}$ & $\mathrm{H}$ & $\mathrm{L}$ & $\mathrm{L}$ & $\mathrm{L}$ & \multirow{6}{*}{ Model unit 2} \\
\hline Staff 6 & $\mathrm{~L}$ & $\mathrm{~L}$ & $\mathrm{H}$ & $\mathrm{H}$ & $\mathrm{L}$ & $\mathrm{H}$ & $\mathrm{L}$ & $\mathrm{L}$ & $\mathrm{L}$ & $\mathrm{L}$ & \\
\hline Staff 7 & M & M & $\mathrm{H}$ & $\mathrm{H}$ & M & M & $\mathrm{H}$ & M & M & $\mathrm{H}$ & \\
\hline Staff 8 & M & $\mathrm{L}$ & $\mathrm{H}$ & $\mathrm{H}$ & $\mathrm{L}$ & $\mathrm{L}$ & $\mathrm{H}$ & $\mathrm{L}$ & $\mathrm{L}$ & $\mathrm{L}$ & \\
\hline Staff 9 & M & M & $\mathrm{H}$ & $\mathrm{H}$ & $\mathrm{L}$ & M & $\mathrm{L}$ & $\mathrm{L}$ & $\mathrm{L}$ & $\mathrm{L}$ & \\
\hline Staff 10 & $\mathrm{~L}$ & M & $\mathrm{H}$ & $\mathrm{H}$ & $\mathrm{L}$ & $\mathrm{L}$ & M & $\mathrm{L}$ & $\mathrm{L}$ & $\mathrm{L}$ & \\
\hline Staff 11 & M & $\mathrm{L}$ & $\mathrm{H}$ & $\mathrm{H}$ & $\mathrm{H}$ & $\mathrm{H}$ & $\mathrm{H}$ & M & $\mathrm{L}$ & $\mathrm{L}$ & \multirow{4}{*}{ Model unit 3} \\
\hline Staff 12 & $\mathrm{~L}$ & $\mathrm{~L}$ & $\mathrm{~L}$ & M & $\mathrm{L}$ & M & $\mathrm{L}$ & M & $\mathrm{L}$ & $\mathrm{L}$ & \\
\hline Staff 13 & $\mathrm{~L}$ & $\mathrm{~L}$ & M & $\mathrm{H}$ & M & M & $\mathrm{L}$ & $\mathrm{L}$ & $\mathrm{L}$ & $\mathrm{L}$ & \\
\hline Staff 14 & $\mathrm{H}$ & $\mathrm{L}$ & M & $\mathrm{H}$ & $\mathrm{H}$ & $\mathrm{H}$ & $\mathrm{H}$ & $\mathrm{M}$ & $\mathrm{L}$ & $\mathrm{L}$ & \\
\hline
\end{tabular}

Figure 4: The overall process of the three model. The level distinction is shown in the bottom, and the model unit distinction is shown on the right.

This section first introduces the subway power supply system in detail from six aspects, focusing on the analysis of China's subway traction power supply system composition and common traction power supply mode. Then, the modeling objective of this paper is pointed out, that is, to establish a traction power supply system model including external power supply, DC traction substation, DC bus, catenary, rail, and ground. The schematic diagram of the proposed model is drawn.

\section{MATLAB-Based Subway Traction Power Supply System Monitoring Test Platform Design}

3.1. Modeling of Metro Traction Power Supply System. There has been a relatively complete power system simulation module in MATLAB. Many existing modules can be used to model traction power supply systems. Therefore, you just need to find the appropriate module from the existing component library and set the relevant parameters. By connecting the modules together, the final model of the traction power supply system is completed. In China's previous metro transport traction power supply system, 12pulse rectifier circuit is often used to reduce the influence of harmonics on the system. But gradually, with the rapid development of power electronics technology in China, researchers found that the equivalent 24-pulse rectifier circuit could better suppress the harmonics, so the 12-pulse rectifier circuit has gradually retired from the stage of history.

The main connection of DC side of metro transport traction power supply system can be divided into double-bus system and single-bus system according to bus form. The double-bus system consists of working bus, standby bus, and bypass switch. There are four feeder lines in the system. The two incoming lines of the bus are equipped with DC circuit breakers. There is service between the working bus and the standby bus. The two-way incoming line of single-bus system generally uses electrical isolation switch or DC circuit breaker, and the return side of rectifier unit generally uses isolation switch. This type of system usually also has a fourway feeder. However, in case of switch failure or overhaul of the system, the normal operation of the system will not be affected by the two connection modes, so the operation of the two different connection modes is guaranteed. Dual-bus bars are safer than single-bus bars, but the cost of a dual bus system is also very high. Taking various factors into consideration, China's metro transport traction power supply system generally adopts a single-bus system, as shown in Figure 5 .

The method used in this paper can be introduced into the system integration algorithm, and both health rate and work efficiency are the highest. Refer to the relevant information in this article to know the line structure in the single-bus system, so this article can establish the equivalent model of the subway DC bus; the model diagram is shown in Figure 6.

The traction grid is the part that provides the power and current path directly to the locomotive load. Traction network is mainly composed of contact network, rail, and return part. Generally speaking, the catenary of subway has two functions. One is the direct supply of DC power to electric locomotives, and the other is direct contact with the pantoon friction. During this process, the catenary can be regarded as a pantograph. When the pantograph is in contact with the power grid, a series of electromagnetic transient phenomena often occur.

Simulate the characteristics of the traction net. Because the focus of this article is the load characteristics and fault characteristics of the traction power supply system, this article must simplify the traction network system, which 


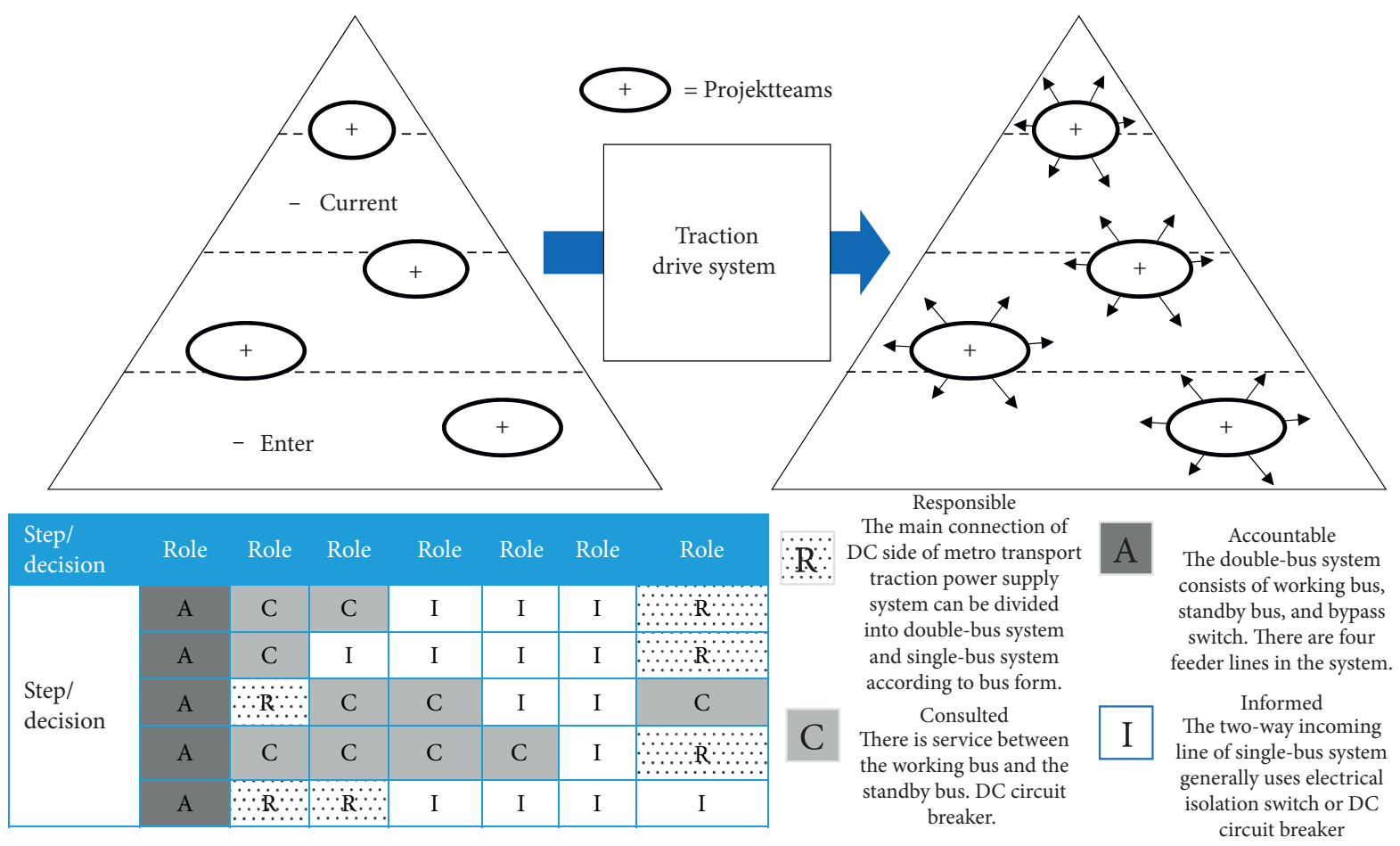

FIGURE 5: Subway transportation traction power supply system. The handle process is on the top, and the specific role for decision-making is shown at the bottom.

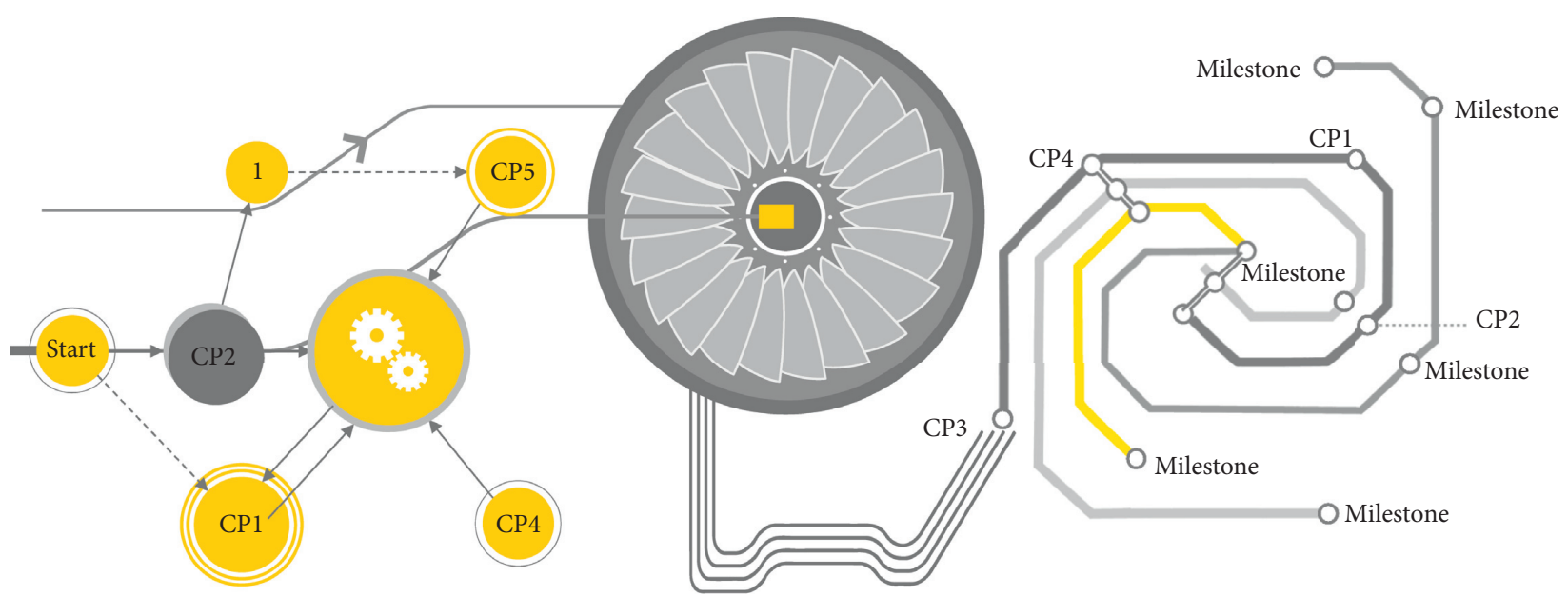

FIgURE 6: The model diagram of the subway DC bus. The process among CP1 CP3 and milestone.

requires this article to ignore the electromagnetic transient phenomenon fuel of the traction network system. Since Chengdu Metro chose the suspended catenary, considering the skin effect after the rail is conductive, the electrical models of the catenary and the rail are different. Here, the models of the catenary and the rail are established separately.

There are transmission line models and cable models in the MATLAB module library and can be used to simulate large-scale power systems. The models of transmission lines can be divided into two types: the equivalent lumped parameter component model and the traveling wave model. Because the model of the line under different power supply modes is different when it is used, and as the length of the power supply arm in the adjacent power supply section is different, the model of the line will also be different. When it is encountered that only the port conditions of the line need to be analyzed, that is, the voltage, current, and power at both ends, usually the distributed parameters of the line can be ignored, and the lumped parameter model can be used to simulate the transmission line. When the line is long, the distributed parameters of the line need to be considered, and the traveling wave model is used to simulate the transmission line.

3.2. Design of the Monitoring Test Platform of the Subway Traction Power Supply System Based on MATLAB. Tracks are part of the normal operation and return of locomotives 
along the subway line. In general, the production material is steel and some other mixtures. Due to the special structure of the rail, its shape is relatively irregular, resulting in the rail permeability becoming very high. When current is applied, severe skin effect will occur inside the rail. Therefore, this article finds that the impedance value of the subway rail is not fixed during the process of electrifying the rail and will change according to the frequency. In-depth study of the simulation modeling of the running rail, and a simplified model of the skin effect of the running rail is given. Here, the rail model used in the reference is used for modeling. The model in this paper uses a fixed time constant orbital equivalent model. The fixed time constant method is a commonly used calculation method in engineering. The focus is on the selection of the time constant. There are two methods usually used: one is to use track DC resistance and power frequency inductance to obtain the fault current time constant; the other is to use the waveform of a typical shortcircuit test to find the equivalent time constant, which is the fitting index. The time constant of the curve: when there are enough short-circuit test samples, the fixed time constant method is used to obtain the short-circuit current curves at different system operation modes and different fault points, so as to obtain a time constant area division and obtain the corresponding track resistance and inductance. Here, this article sets the distance between two adjacent substations of Chengdu Metro to 4 kilometers, the equivalent resistance value of the rail is set to 0.032 ohms per kilometer, and the equivalent inductance value of the rail is set to $1.79 \mathrm{mH}$ per kilometer. Set the equivalent capacitance value of the rail to $0.05 \mathrm{~S}$ per kilometer. According to the above parameters, the test trend of the actual test platform model can be obtained, as shown in Figure 7.

On the basis of this rail model, this article establishes the electrical model of the Earth and return line. Here, we choose to adopt a four-layer model. In order to better equate the distribution of stray currents and the changes in the potential of the rail, this article generally has two models to choose from when the rail and the ground are equivalent. The simpler one is the three-layer network model, which generally only considers the catenary, steel rails, and the ground, so the model is relatively simple, usually by connecting many n-type unit circuits in parallel to complete the modeling. Another more complicated one is the four-layer network model, which adds buried metals to the model network on the basis of the three-layer network. In general, in this model, this paper takes the recirculation network formed by the rail and ground network and the buried metal and ground network.

The formed recirculation nets are all simulated with type circuits. In general, the unit division of type 2 circuits in this article is 100 to 200 meters. Since in the actual subway line there are indeed some stray current return networks formed by the steel bars of other buildings along the subway line, the model of the four-layer network will also be more realistic, and modeling using this model will make the built model more reasonable $[19,20]$.

From the above four-layer network structure diagram, we can see that the steel rails and the buried metal mesh are connected to the ground by some longitudinal unit circuits. Moreover, this article can also see that each unit is composed of the longitudinal resistance of the rail and the buried metal mesh and the transition resistance of the rail, the buried metal mesh, and the ground. However, because in actual lines the rails and buried metal meshes in different sections and sections are different, in fact, the longitudinal direction and the size of the transition resistance of each unit are different. In order to simplify the model here, the transition resistance of each unit has been omitted in the figure. And here, this article idealizes the model, assuming that the electrical parameters of the entire line are exactly the same, and the distance between each unit is 100 to 200 meters. Then, this article can build rail and ground impact-uncertainty matrix as shown in Figure 8.

This section establishes the model of each part of subway traction power supply system on the MATLAB platform. Firstly, the external power supply model is established. Then, the working principle of 24-pulse rectifier transformer is analyzed in detail, and the model of traction substation is established by using the transformer and rectifier module in MATLAB. Then, the DC bus model of subway traction power supply system is established. Finally, the PI model in the component library is used to build the subway catenary model, and the four-layer ground network structure is used to build the track and ground model. It lays a good foundation for the simulation of subway traction power supply system under subsequent conditions. The health assessment data are shown in Table 1:

\section{Health Assessment of Subway Traction Power Supply System}

4.1. Hierarchical Immune Health Assessment Mechanism of Subway Traction Power Supply System. Hierarchical analysis model of traction power supply system: subway traction power supply system is a large and complex system, which is suitable for hierarchical processing to solve multiattribute decision-making problems. According to the hierarchy division, the analytic hierarchy process (AHP) model of traction power supply system is established, to determine the membership relationship between the elements in each level, compare the elements of the same level in pairs, and obtain a comparison of the importance of $n$ different elements of the same level relative to the corresponding elements of the previous level. The order judgment matrix is constructed as a pair comparison matrix. In the process of establishing a paired comparison matrix, the order of the matrix and the difference of subjective thinking will have a certain impact on the matrix established, so the initial relative weight value needs to be uniformly checked.

Since a fuzzy set can be characterized as the sum of all elements with a certain property to different degrees, the membership function can reflect the degree to which these elements belong to the fuzzy set, and its value is between 0 and 1 ; the greater the value of the membership function, the higher the degree that the index belongs to this fuzzy set. In this paper, the equal division method is used to determine the membership value within the fuzzy distribution value interval, which is convenient for the automatic processing of computer 


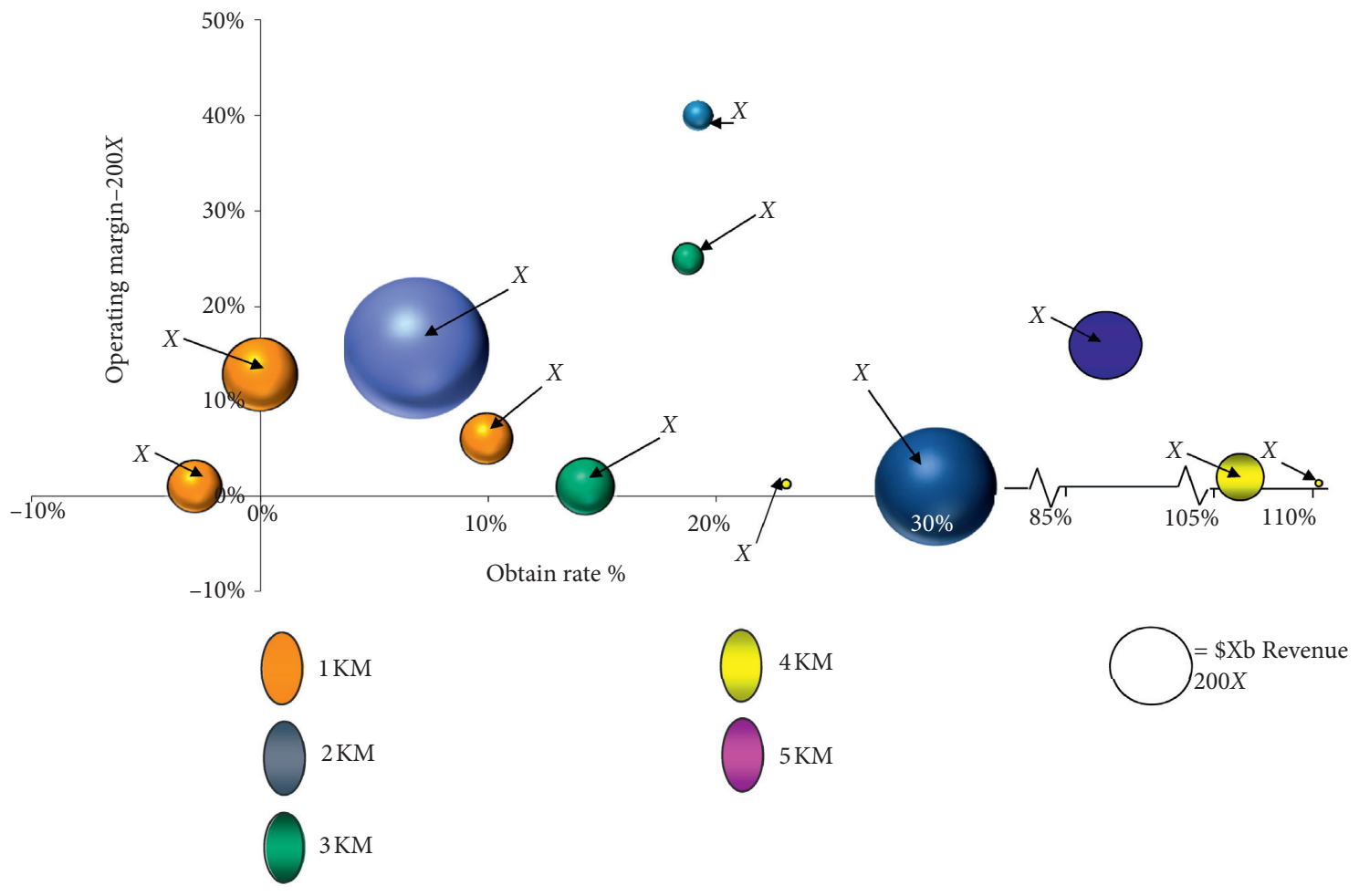

FIgURE 7: The test obtain rate of the actual test platform model. The interval is shown on the right.

programming, and the algorithm has little effect on the evaluation accuracy. According to the physical meaning of the health indicators, combined with the analysis of the evaluation system, two fuzzy distribution forms of benefit type (the larger the better) and cost type (the smaller the better) are considered. According to the design standards, inspection specifications, and operating characteristics of each component of the traction power supply system, the value range of the set index is $(a, b)$, and three equal points are inserted in this interval to divide it into four equal parts. Because the objective correction coefficient is introduced to correct the initial relative weight, the influence of subjective factors in determining the initial relative weight is effectively eliminated. The comprehensive weight obtained in the health assessment not only absorbs the advantages of subjective convenience and efficiency, but also pays attention to objectivity without loss of accuracy and rigor combines the advantages of the two organically. The overall process and data of this method for the health assessment of the subway traction power supply system is shown in Figure 9. It can be seen from Figure 9 that this method achieves systematic analysis and evaluation by obtaining health values from component level to subsystem level to system level step by step.

4.2. Examples of Health Assessment of Subway Traction Power Supply System. At the system level, the subway traction power supply system $S$ considers that the main substation, traction substation, and step-down substation can be mutually backup through cross-zone power supply, while the catenary and medium-voltage ring network have no backup. It has been verified that the consistency check meets the requirements. If only the health status of the aforementioned traction substation TS1 is considered, it is found that it is in a healthy state, because other adjacent traction substations and equipment connected to the medium-voltage ring network are backups for each other and can be replaced by cross-zone power supply, so it has little effect on the health of the system.

If only the health status of the aforementioned catenary is considered, the health value of the subway traction power supply system $S$ is $H s=0.9948$, which is in a healthy state, but the health value is slightly smaller than when only the health state of the traction substation is considered, because the catenary subsystem has a larger influence weight on the system health value than the traction substation subsystem, which is consistent with the feature that the catenary has no backup. If the health status of the traction substation TS1 and the catenary OC are taken into account at the same time, the health value of the subway traction power supply system $S$ is $H s=0.9902$, which is still in a healthy state, but its health value is further reduced. The change trend of the subway health assessment value with the health status is shown in Figure 10. 


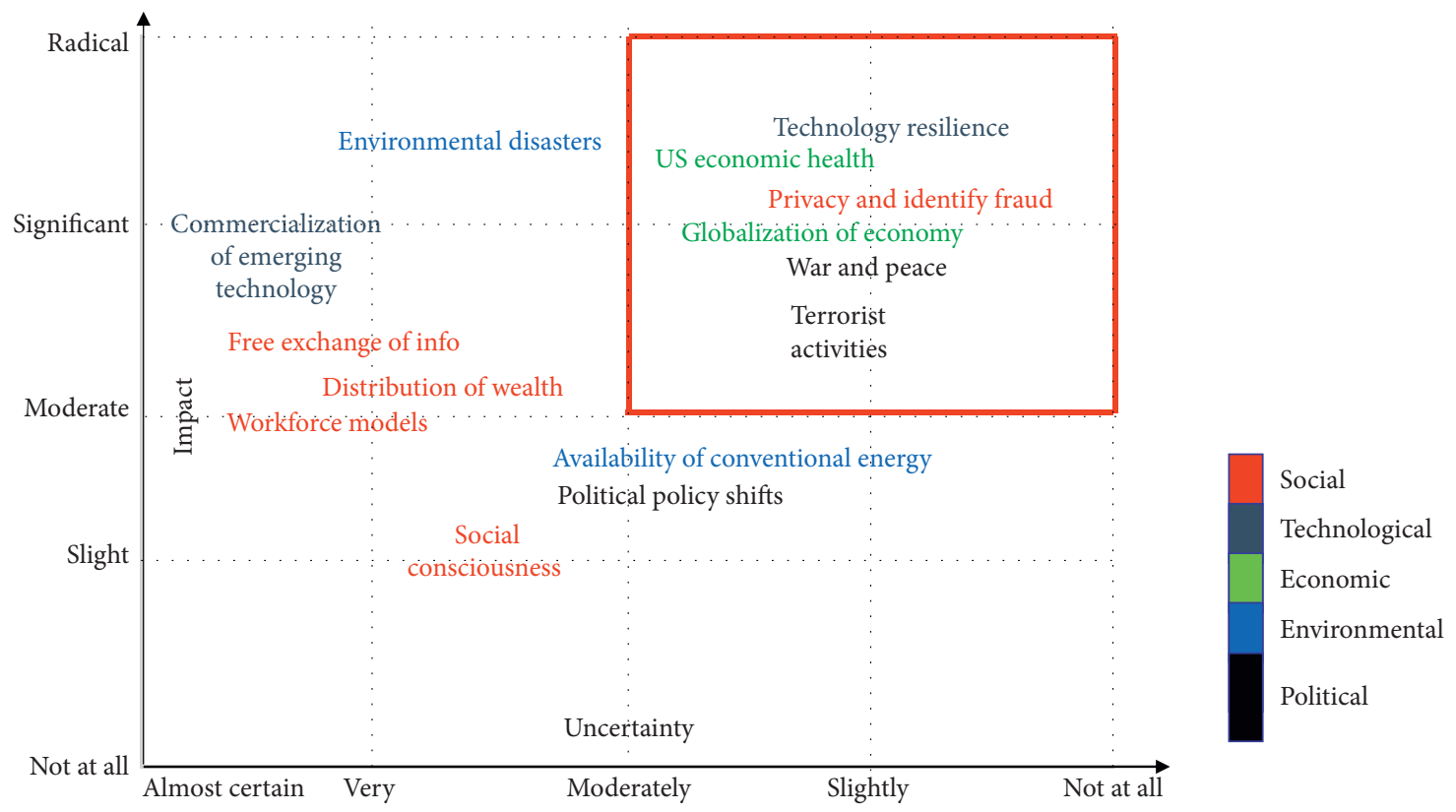

FIGURE 8: Rail and ground impact-uncertainty matrix. The impact type is shown on the right.

TABLE 1: The health assessment data.

\begin{tabular}{lccc}
\hline Assessment type & Health index & Conversion efficiency (\%) & Health data ratio (\%) \\
\hline Old subway system & 0.512 & 45.70 & 43.10 \\
New subway system & 0.764 & 77.80 & 82.00 \\
\hline
\end{tabular}

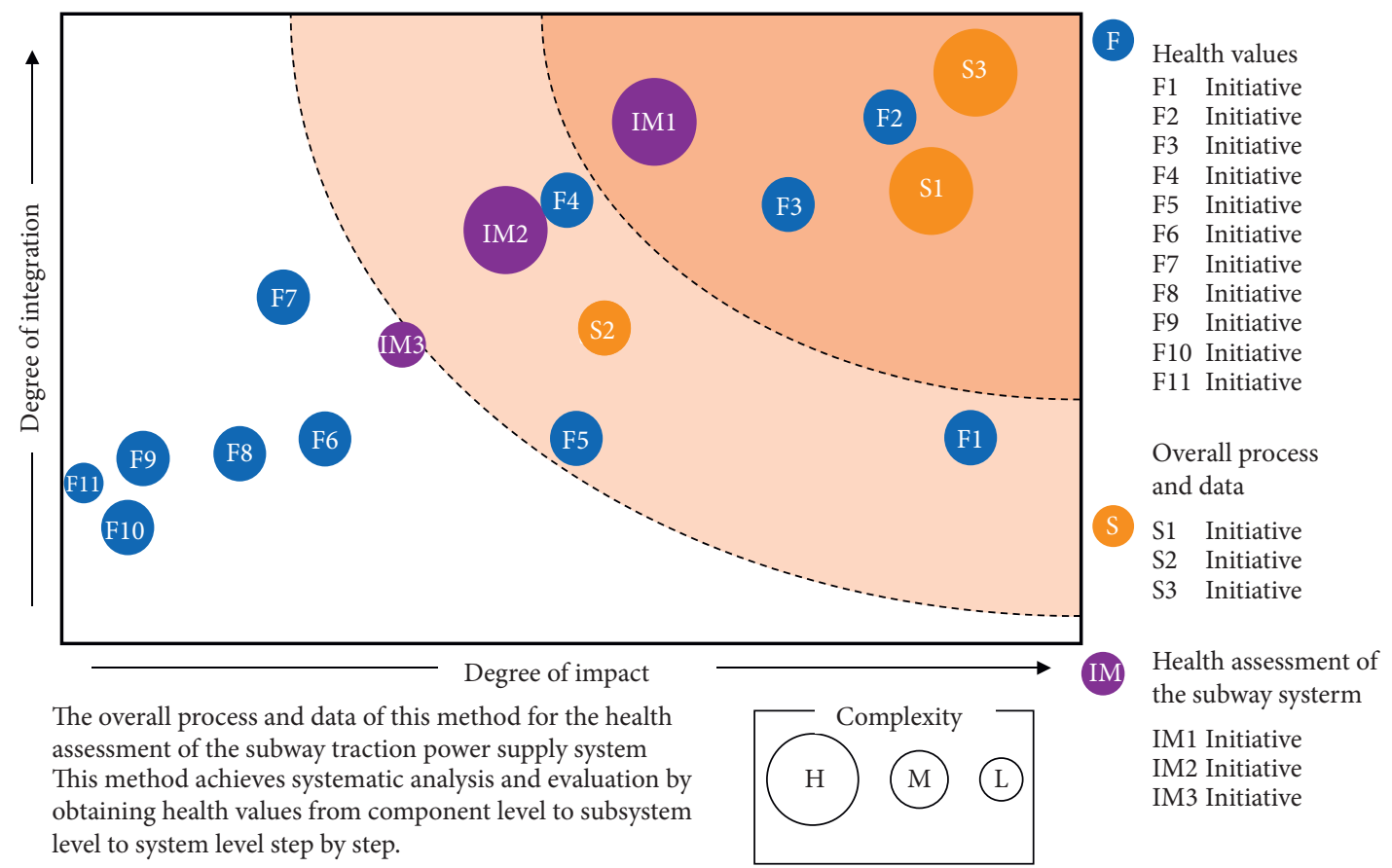

FIGURE 9: Method achieves systematic analysis and evaluation by obtaining health values. The degree of color means the importance level of all the impact. 


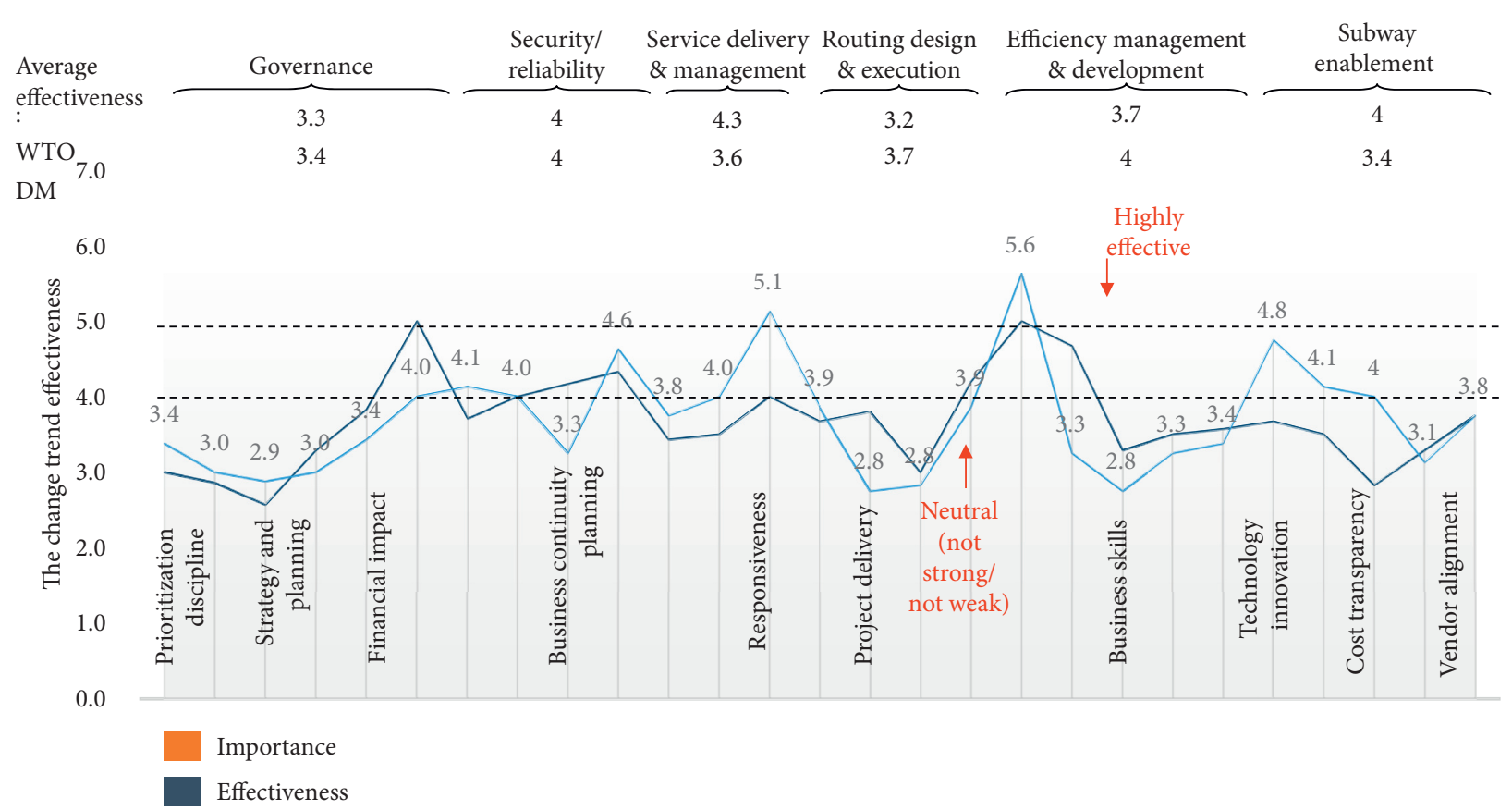

Figure 10: The change trend of the subway health assessment value with the health status. Highly effective and neutral are shown by the red arrow.

\section{Conclusion}

The design of monitoring and testing platform and health assessment of subway traction power supply system are helpful for traction power supply system to provide accurate and timely health information of traction power supply system, dynamic identification of safety equipment affected by function degradation, determination of maintenance subsystems and components, and corresponding maintenance strategies. In this paper, the AHP model of subway traction power supply system is established, the system layer, subsystem layer, component layer, and index layer are divided, and the immune monitoring system of subway traction power supply system is constructed. On this basis, the initial relative weight is based on paired comparison matrix, fuzzy statistics method is adopted, and the concept of fuzzy membership degree is introduced. The purpose is to obtain the correction coefficient to modify the initial relative weight and eliminate the subjective influence on the weight calculation results, and at the same time, the difference in thinking is combined with the advantages of subjective and objective. Bilateral power supply types of metro traction power supply system, for example, step by step, traction substation, catenary traction rectifier transformer, and the actual analysis of traction power supply system, realized the subway traction power supply system of the health of the comprehensive and quantitative and systemic evaluation; the reliability analysis and fault early warning forecast, risk assessment, safety, and maintenance policy research laid the foundation. In the future direction, this paper can consider the electromagnetic transient phenomenon of the pantograph system when building the model of traction power supply system, which can make the model established in this paper more accurate. In this paper, the fault occurring in different sections and the short circuit of the locomotive under different working conditions can be further considered, which can make the simulation results closer to the real value. In addition to the disadvantages listed above, it is an important research direction for modeling and simulation of subway traction power supply system to make relevant research toward a more reasonable treatment of the influence of transition resistance on the system.

\section{Data Availability}

No data were used to support this study.

\section{Consent}

Informed consent was obtained from all individual participants included in the study references.

\section{Conflicts of Interest}

The authors declare that there are no conflicts of interest.

\section{Acknowledgments}

This paper was supported by an open fund from JiangSu Research and Development Center of Rail Transportation Control Engineering Technology (no. KFJ2007) and the Natural Science Foundation of the Jiangsu Higher Education Institutions of China (no. 19KJB560018).

\section{References}

[1] G. Xing and W. U. Long, "Study on rail potential of metro traction power supply system," Supply System, vol. 17, no. 2, pp. 45-122, 2019. 
[2] W. Weiying and W. Mingliang, "Method for improving power quality of metro traction power supply system with PV integration," PV Integration, vol. 1, no. 1, pp. 1682-1685, 2017.

[3] X. Song, J. He, T. Yip, B. Kirby, and X. Yang, "Research of fault location method for metro traction power supply system," Lecture Notes in Electrical Engineering, vol. 288, no. 1, pp. 137-146, 2014.

[4] V. A. Grechishnikov, "Calculation of metro traction power supply considering partial recuperation currents outputted by Rusich-type railway cars," Russian Electrical Engineering, vol. 5, no. 7, pp. 112-155, 2017.

[5] M. Toge, H. Igarashi, and Y. Shimizu, "Introducing two effective utilization systems of regenerating power in traction power supply system on Tokyo metro," IEEE, vol. 43 , no. 1 , pp. 288-293, 2017.

[6] Z. Ma, J. Jiang, S. Liu, Z. Wang, and M. Gong, “The design of traction power battery system for dual power urban rail metro," EITRT, vol. 2, no. 1, pp. 201-233, 2013.

[7] Y. H. Li, X. J. Tian, and X. Q. Li, "Nonlinear second-order model and equilibrium point characteristic analysis of DC traction power supply system," Isrn Applied Mathematics, vol. 1, pp. 221-228, 2013.

[8] M. T. Lazarescu, "Design and field test of a WSN platform prototype for long-term environmental monitoring," Sensors, vol. 47, no. 3, pp. 201-231, 2015.

[9] M. Adolfo, S. Roberto, P. Mario et al., "Proof-of-concept design and development of an EN13606-based electronic health care record service," Journal of the American Medical Informatics Association, vol. 1, no. 1, pp. 118-129, 2019.

[10] C. J. Licskai, T. W. Sands, and M. Ferrone, "Development and pilot testing of a mobile health solution for asthma selfmanagement: asthma action plan smartphone application pilot study," Canadian Respiratory Journal of the Canadian Thoracic Society, vol. 20, no. 4, p. 301, 2016.

[11] I. S. Bessa, "Theoretical studies on kinetics, mechanism and thermochemistry of gas-phase reactions of HFE-449mec-f with $\mathrm{OH}$ radicals and $\mathrm{Cl}$ atom: a subchronic (180-day) oral toxicity study of ethyl tertiary-butyl," Air Quality Atmosphere \& Health, vol. 7, no. 4, pp. 415-420, 2014.

[12] C. Ndwiga, H. Birungi, C. C. Undie, H. Weyenga, and J. Sitienei, "Feasibility and effect of integrating tuberculosis screening and detection in postnatal care services: an operations research study," BMC Health Services Research, vol. 13, no. 1, pp. 99-100, 2013.

[13] O. Justin, B. Matthew, C. Lophina et al., "Evaluation of the Xpert MTB/RIF assay at a tertiary care referral hospital in a setting where tuberculosis and HIV infection are highly endemic," Clinical Infectious Diseases, vol. 55, no. 9, pp. 1171-1178, 2012.

[14] S. Qiu and J. Wang, "Application of sensory evaluation, HSSPME GC-MS, E-nose, and E-tongue for quality detection in citrus fruits," Journal of Food Science, vol. 80, no. 10, pp. 2296-3047, 2015.

[15] A. Sobrinho, L. D. Silva, A. Perkusich, M. E. Pinheiro, and P. Cunha, "Design and evaluation of a mobile application to assist the self-monitoring of the chronic kidney disease in developing countries," BMC Medical Informatics \& Decision Making, vol. 18, no. 1, p. 7, 2018.

[16] K. Tanaka, S. Irikoma, O. Malafaia et al., "Evaluation of anatomopatho," International Journal of Digital Content Technology \& its Applications, vol. 6, no. 21, pp. 550-555, 2017.

[17] A. Lee, M. Sandvei, H. C. Asmussen et al., "The development of complex digital health solutions: formative evaluation combining different methodologies," Jmir Research Protocols, vol. 7, no. 7, p. 165, 2018.

[18] M. Chen, J. Yang, J. Zhou, Y. Hao, J. Zhang, and C.-H. Youn, "5G-smart diabetes: toward personalized diabetes diagnosis with healthcare big data clouds," IEEE Communications Magazine, vol. 56, no. 4, pp. 16-23, 2018.

[19] J. Wang, A. Liu, T. Yan, and Z. Zeng, "A resource allocation model based on double-sided combinational auctions for transparent computing," Peer-to-Peer Networking and Applications, vol. 11, no. 4, pp. 612-633, 2018.

[20] X. Feng, H. Zhang, Y. Ding, Z. Liu, H. Peng, and B. Xu, "A review study on traction energy saving of rail transport," Discrete Dynamics in Nature and Society, vol. 2013, no. 1, 1617 pages, Article ID 156548, 2013. 